\title{
Dynamical Systems Approach to Magnetised Cosmological Perturbations
}

\author{
Stacey Hobbs* and Peter K. S. Dunsby ${ }^{\dagger}$ \\ Department of Mathematics and Applied Mathematics, University of Cape Town, Rondebosch 7701, Cape Town, South Africa.
}

(November 3, 2018)

\begin{abstract}
Assuming a large - scale homogeneous magnetic field, we follow the covariant and gauge-invariant approach used by Tsagas and Barrow to describe the evolution of density and magnetic field inhomogeneities and curvature perturbations in a matter-radiation universe. We use a two parameter approximation scheme to linearize their exact non-linear general-relativistic equations for magneto-hydrodynamic evolution. Using a two-fluid approach we set up the governing equations as a fourth order autonomous dynamical system. Analysis of the equilibrium points for the radiation dominated era lead to solutions similar to the super-horizon modes found analytically by Tsagas and Maartens. We find that a study of the dynamical system in the dust-dominated era leads naturally to a magnetic critical length scale closely related to the Jeans Length. Depending on the size of wavelengths relative to this scale, these solutions show three distinct behaviours: large-scale stable growing modes, intermediate decaying modes, and small-scale damped oscillatory solutions.
\end{abstract}

\section{INTRODUCTION}

Magnetic fields play an important role in our Universe. They appear on all scales, from the solar system, through interstellar and extra-galactic scales, to intracluster scales of several Mpc. Although magnetic field inhomogeneities have not yet been observed on scales as large as those exhibited by Cosmic Microwave Background anisotropies, it is natural to expect that magnetic fields exits on such scales [1], and that they could play a role in the formation of large-scale structure.

Magnetic fields in intergalactic space are notoriously invisible. In the early 1950's the discovery of synchrotron radiation from the interstellar medium of the Milky Way led to the realization that it possessed a magnetic field. In 1957 Bolton and Wild [2] suggested that the Zeeman splitting of a radio transition should be observable in the interstellar gas. This provided a direct way of measuring the strength of this uniform magnetic field. That almost ten years passed between this suggestion and the first detections of this effect is an indication of the technical difficulty such measurements posed at the time [3]. Since then, advances in observational techniques have produced firmer estimates of magnetic field strengths in interstellar and intergalactic space.

We now know that a large scale, organized magnetic field fills the disk of the Milky Way. Studies of external galaxies indicate that all disc galaxies are permeated by large scale magnetic fields [4], and that $\mu G$ level fields are common in spiral galaxy discs and halos. Strengths of magnetic fields in intergalactic gas in 'normal' galaxy clusters have been measured using Faraday Rotation Measures (RM) combined with $\mathrm{X}$-ray data. This gives typical field strengths for these fields of between 2 and $6 \mu G$, [5], which is comparable to the field strengths in the denser interstellar medium in our galaxy. In 1993, Taylor and Perley [6] discovered that these field strengths were exceeded by those in some less common, but more dense cooling flow clusters, where the field strength can be as high as $30 \mu G$. These fields show ordered components on super-galactic scales [5].

Much of our local universe consists of voids, regions containing very little baryonic matter. As yet, the weaker fields within these voids, which may be relics of true cosmic primordial fields, remain unmeasured. Primordial and protogalactic magnetic fields represent the largescale fields which could play a role in structure formation. There are at present over thirty theories about the origin of cosmic magnetic fields at galactic and intergalactic scales. Battaner \& Lesch [1] look at astrophysical arguments to examine these models. These can be divided into four main categories, based on when the fields are generated, namely: a) during inflation, b) in a phase transition after inflation, c) during the radiation dominated era, and d) after recombination. It is the large scale fields which were produced during inflation which are most likely to have implications for structure formation.

Investigating the effect of magnetic fields on structure formation is not a recent endeavour. In 1971, Ruzmaikin \& Ruzmaikina [7] gave a Newtonian analysis of the growth of density perturbations in a perfectly con-

\footnotetext{
*E-mail: stacey@maths.uct.ac.za

${ }^{\dagger}$ E-mail: peter@vishnu.mth.uct.ac.za
} 
ducting medium with magnetic fields. Wasserman 8] considered magnetic influence on galaxy formation, and angular momentum. Kim et al [9] extend this by including the back-reaction of the fluid on to the field, and as a result, derived a magnetic Jeans length. Battaner et al. [10] present relativistic analysis of the evolution of magnetic fields and their influence on density inhomogeneities in a radiation dominated universe, while Jedamzik et al. and Subramanian \& Barrow [11] have considered magnetic dissipative effects at recombination.

More recently Tsagas and Barrow 12 14 have developed a covariant and gauge-invariant approach to the analysis of magnetized density perturbations, in a universe model containing a perfect fluid. Using the covariant gauge-invariant formalism of Ellis and Bruni [15], they derived a set of exact non-linear equations for general-relativistic magneto-hydrodynamic evolution. On linearizing these equations around a flat Friedman-Robertson-Walker (FRW) model, they were able to identify the general relativistic corrections to earlier Newtonian work, including the existence of a magnetocurvature coupling.

Tsagas and Maartens [16] extended these results, considering also shape distortion effects due to the fields as well as density and rotational perturbations They found explicit solutions to the perturbation equations for the radiation and dust eras, as well as pure-magnetic density perturbations. They also identified and analysed other sources of the magnetic effects.

When studying the evolution of density perturbations, the dynamics of the perturbed universe can be represented by an autonomous dynamical system, described by a set of coupled differential equations for the gaugeinvariant perturbation variables 17 19. This allows the stability behaviour of the model to be investigated with relative ease, with no need to solve for the background variables. Furthermore, stationary points of the dynamical system correspond to exact or approximate analytic solutions of the linearised perturbation equations, thus providing a useful tool for obtaining solutions in cosmologically interesting situations.

This paper is outlined as follows. In section III, we set up the evolution equations of the density and magnetic field inhomogeneities, and the curvature perturbations. We discuss in detail the approximations and linearisation procedure in sections [V] \& V and identify an extra term in the linearized propagation equation for the spatial curvature $\mathcal{K}$. In section VII, we set up a five dimensional autonomous dynamical system equivalent to the linearized propagation equations derived in section III - these equations give a detailed description of the full phase-space of solutions for perturbation dynamics in a magnetized dust - radiation universe. The analysis and discussion of this system, with particular emphasis on the three dimensional invariant sets representing the initial radiation and final dust dominated states are given in section VIID. Finally, conclusions are given in section VIII.

\section{DEFINING VARIABLES}

\section{A. Spacetime Splitting}

For all of the following equations, we will use standard units defined by $8 \pi G=c=1$. In cosmology, the average velocity of matter at each spacetime event defines a unique 4 - velocity vector: $u^{a}=\frac{d x^{a}}{d \tau}$ with $u_{a} u^{a}=-1$. The fundamental fluid-flow lines defined by this vector field are a congruence of worldlines, carrying the fundamental observers. The projection tensor $h_{a b}=g_{a b}+u_{a} u_{b}$, (with $h^{a}{ }_{c} h_{b}^{c}=h_{b}^{a}$, and $h^{a}{ }_{a}=3$ ) projects into the local rest spaces of comoving observers.

The covariant derivative of any tensor $T^{a b}{ }_{c d}$ may be split into a time derivative along the fluid flow,

$$
\dot{T}^{a b}{ }_{c d}=u^{e} \nabla_{e} T^{a b}{ }_{c d}
$$

and a totally projected covariant derivative orthogonal to the fluid flow [20]

$$
\tilde{\nabla}_{e} T^{a b}{ }_{c d}=h^{a}{ }_{f} h_{g}^{b} h^{p}{ }_{c} h^{q}{ }_{d} h^{r}{ }_{e} \nabla_{r} T^{f g}{ }_{p q},
$$

with total projection on all free indices. It is worth noting that if $u^{a}$ has non-zero vorticity, $\tilde{\nabla}$ is not a proper 3 dimensional covariant derivative. Angle brackets denote orthogonal projection of vectors $V^{\langle a\rangle}=h^{a}{ }_{b} V^{b}$, and the projected symmetric trace-free (PSTF) part of tensors $T^{\langle a b\rangle}=\left[h^{(a}{ }_{c} h^{b)}{ }_{d}-\frac{1}{3} h^{a b} h_{c d}\right] T^{c d}$ [21].

Following [16], we can define a covariant spatial divergence and curl that generalize the Newtonian operators to curved spacetime:

$$
\begin{gathered}
\operatorname{div} V=\tilde{\nabla}^{a} V_{a}, \quad(\operatorname{div} T)_{a}=\tilde{\nabla}^{b} T_{a b}, \\
\operatorname{curl} V_{a}=\varepsilon_{a b c} \tilde{\nabla}^{b} V^{c}, \quad \operatorname{curl} T_{a b}=\varepsilon_{c d(a} \tilde{\nabla}^{c} T_{b)}{ }^{d}
\end{gathered}
$$

where $\varepsilon_{a b c} \equiv \eta_{a b c d} u^{d}$ is the projected covariant permutation tensor.

\section{B. Kinematic Quantities}

The projection tensor, together with the velocity 4vector, split the covariant derivative of $u_{a}$ into irreducible basic kinematic quantities [20]:

$$
\begin{aligned}
\nabla_{b} u_{a} & =-u_{b} \dot{u}_{a}+\tilde{\nabla}_{b} u_{a} \\
& =-u_{b} \dot{u}_{a}+\frac{1}{3} \Theta h_{a b}+\sigma_{a b}+\omega_{a b}
\end{aligned}
$$

where $\dot{u}_{b}=\nabla_{a} u_{b} u^{a}, \Theta=\tilde{\nabla}_{a} u^{a}, \sigma_{a b}=\tilde{\nabla}_{\langle a} u_{b\rangle}$ and $\omega_{a b}=$ $\tilde{\nabla}_{[b} u_{a]}$. These are, respectively, the 4 -acceleration, the expansion scalar, the shear tensor and the vorticity tensor. The expansion scalar is used to introduce a representative length scale $a$ along the observers' worldline which in turn defines the Hubble expansion rate, $\frac{\dot{a}}{a}=\frac{1}{3} \Theta=H$. 
The evolution of the expansion scalar is described by Raychaudhuri's equation:

$$
\dot{\Theta}-A+2\left(\sigma^{2}-\omega^{2}\right)+\frac{1}{3} \Theta^{2}+\frac{1}{2}\left(\rho+3 p+B^{2}\right)=0,
$$

where $A=\nabla^{a} \dot{u}_{a}, \sigma^{2}=\frac{1}{2} \sigma^{a b} \sigma_{a b}$ and $\omega^{2}=\frac{1}{2} \omega^{a b} \omega_{a b}$ (see 20 for details).

Local curvature is described by the Ricci tensor $R_{a b}$ while non- local tidal forces and gravitational radiation are described by the 'electric', $E_{a b}$ and 'magnetic', $H_{a b}$ parts of the Weyl tensor. These are a pair of symmetric, traceless and completely spacelike second order tensors, defined by:

$$
\begin{gathered}
E_{a b}=E_{\langle a b\rangle}=C_{a c b d} u^{c} u^{d}, \\
H_{a b}=H_{\langle a b\rangle}=\frac{1}{2} \varepsilon_{a c d} C_{b e}^{c d} u^{e} .
\end{gathered}
$$

\section{The Electromagnetic Field}

The electromagnetic field is represented by the Maxwell tensor $F_{a b}=F_{[a b]}$. As seen by an observer with 4 - velocity $u_{a}$, the field tensor splits into an electric $E_{a}$ and a magnetic $B_{a}$ part (see [20] for details):

$$
\begin{gathered}
E_{a}=F_{a b} u^{b}=-F_{a b} u^{a}, \\
B_{a}=\frac{1}{2} \varepsilon_{a c d} F^{c d},
\end{gathered}
$$

where $E_{a} u^{a}=0$, and $B_{a} u^{a}=0$.

\section{EQUATIONS}

\section{A. Maxwell's Equations}

Using the above decomposition, Maxwell's equations can be written as follows:

$$
\nabla_{b} F^{a b}=J^{a}
$$

and

$$
\nabla_{[c} F_{a b]}=0
$$

where $J^{a}$ is the 4 -current which generates the electromagnetic field and obeys the conservation law $\nabla_{a} J^{a}=0$.

These equations can be split into spatial and temporal parts using the notation defined above:

$$
\begin{gathered}
\operatorname{div} E+2 \omega^{a} B_{a}=q \\
\sigma^{a b} E_{b}+\varepsilon^{a b c} \omega_{c} E_{b}-\frac{2}{3} \Theta E^{a}+\varepsilon^{a b c} \dot{u}_{b} B_{c} \\
+\operatorname{curl} B^{a}=\dot{E}^{<a>}+j^{a}
\end{gathered}
$$

$$
\operatorname{div} B=2 \omega^{a} E_{a}
$$

and

$$
\begin{gathered}
\sigma^{a b} B_{b}-\varepsilon^{a b c} \omega_{b} B_{c}-\frac{2}{3} \Theta B^{a}-\varepsilon^{a b c} \dot{u}_{b} E_{c} \\
-\operatorname{curl} E^{a}=\dot{B}^{<a>} .
\end{gathered}
$$

The 4 -current $J^{a}$ enters into equations (12) and (13) through the charge density $q=-J^{a} u_{a}$ and the projected current $j^{a}=J^{<a>}$. The vorticity vector $\omega_{a}$ is related to the vorticity tensor via $\omega_{a b} \equiv \varepsilon_{a b c} \omega^{c}$.

\section{B. Gauge - invariant Variables}

Following [16] and [12], the key covariant and gaugeinvariant variables describing inhomogeneity in the fluid and the magnetic field are the comoving spatial gradients of the energy density $\rho$, the expansion $\Theta$ and the field density $B^{2}$ :

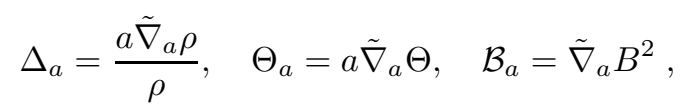

where $B^{2}=B_{a} B^{a}$. Other basic variables which appear in the exact equations are the spatial gradient of the pressure, $Y_{a}=\tilde{\nabla}_{a} p$, the anisotropic pressure generated by the magnetic field, $\Pi_{a b}=\frac{1}{3} B^{2} h_{a b}-B_{a} B_{b}$, and the comoving spatial gradient of the field vector: $B_{a b}=a \tilde{\nabla}_{b} B_{a}$.

\section{Medium and Propagation Formulae}

The matter description under consideration is a mixture of dust and radiation which share the same 4velocity $u^{a}$, interacting only through gravity. In general, the evolution equations for density perturbations couple to an entropy evolution equation through the equation of state $p=p(\rho, s)$, where $s$ is the entropy density. However, since entropy perturbations are only important on very small scales, we will assume adiabatic perturbations at all times, thus ignoring any entropy contributions 22 .

The total fluid equations take the same form as those for a perfect fluid of infinite conductivity. The infinite conductivity approximation allows the electric field to be omitted from Maxwell's Equations while spatial currents are preserved [12].

In this case, Maxwell's equations (12) - (15) generate three constraints 16,

$$
\begin{gathered}
\omega^{a} B_{a}=\frac{1}{2} q, \\
\operatorname{curl} B^{a}=\varepsilon^{a b c} B_{b} \dot{u}_{c}+j^{a}, \\
\operatorname{div} B=0
\end{gathered}
$$

and one propagation equation

$$
\dot{B}^{<a>}=\sigma^{a b} B_{b}+\varepsilon^{a b c} B_{b} \omega_{c}-\frac{2}{3} \Theta B^{a} .
$$




\section{Conservation Equations}

Since we are only considering adiabatic perturbations, we only need the total fluid equations. The energy and momentum density conservation equations are respectively 12]:

$$
\dot{\rho}+\rho(1+w) \Theta=0
$$

and

$$
\rho\left(1+w+\frac{2 B^{2}}{3 \rho}\right) \dot{u}_{a}+Y_{a}-\frac{2}{a} B_{[a b]} B^{b}+\dot{u}^{b} \Pi_{b a}=0,
$$

where $w=\frac{p}{\rho}$. Ignoring entropy perturbations, the pressure can be written as a function of the energy density only, i.e. $p=p(\rho)$. It follows that the relationship between $Y_{a}$ and $\Delta_{a}$ is given by

$$
a Y_{a}=\rho c_{s}^{2} \Delta_{a}
$$

where $c_{s}^{2}=\frac{d p}{d \rho}=\frac{\dot{p}}{\dot{\mu}}$ is the speed of sound in the fluid.

\section{E. Exact Propagation Equations for the Inhomogeneity Variables}

The propagation equations for the various spatial gradients are given in 12]. They are, firstly, the equation for the comoving fractional spatial gradient of the energydensity:

$$
\begin{aligned}
\dot{\Delta}_{<a>} & =w \Theta \Delta_{a}-\left(\sigma^{b}{ }_{a}+\omega^{b}{ }_{a}\right) \Delta_{b}-(1+w) \Theta_{a} \\
& -\frac{2 \Theta}{\rho} B_{[a b]} B^{b}+\frac{2 a \Theta B^{2}}{3 \rho} \dot{u}_{a}+\frac{a \Theta}{\rho} \dot{u}^{b} \Pi_{b a},
\end{aligned}
$$

secondly, the equation that governs the spatial gradient of the expansion:

$$
\begin{aligned}
\dot{\Theta}_{<a>} & =-\frac{2 \Theta}{3} \Theta_{a}-\left(\sigma^{b}{ }_{a}+\omega^{b}{ }_{a}\right) \Theta_{b}-\frac{1}{2} \rho \Delta_{a}+B_{b a} B^{b} \\
& -3 B_{[a b]} B^{b}+a \mathcal{R} \dot{u}_{a}+\frac{3}{2} a \dot{u}^{b} \Pi_{b a}+a A_{a} \\
& -2 a \tilde{\nabla}_{a}\left(\sigma^{2}-\omega^{2}\right)
\end{aligned}
$$

where $A_{a}=h_{a}{ }^{b} A_{; b}=\tilde{\nabla}_{a} A, A=\tilde{\nabla}^{a} \dot{u}_{a}$ and

$$
\mathcal{R}=\frac{1}{2} K+A-3\left(\sigma^{2}-\omega^{2}\right)
$$

with

$$
K=\rho+\frac{1}{2} B^{2}-\frac{1}{3} \Theta+\sigma^{2}-\omega^{2}
$$

representing the 3 -Ricci scalar of the observers instantaneous rest space.

\footnotetext{
${ }^{1}$ Spatial flatness is necessary for the 3 -Ricci scalar to be gauge - invariant.
}

Finally, the equation describing the evolution of the orthogonal spatial gradient of the magnetic field is given by:

$$
\begin{aligned}
a^{-2} h_{a}{ }^{e} h_{b}{ }^{p}\left(a^{2} B_{e p}\right)^{*} & =-B_{a e}\left(\sigma^{e}{ }_{b}+\omega^{e}{ }_{b}\right)+\left(\sigma_{a}{ }^{e}+\omega_{a}{ }^{e}\right) B_{e b} \\
& -\frac{2}{3} B_{a} \Theta_{b}+2 a B_{e} \sigma^{e}{ }_{[a} \dot{u}_{b]} \\
& -2 a B_{e} \omega^{e}{ }_{(a} \dot{u}_{b)}+a B^{e} \tilde{\nabla}_{b}\left(\sigma_{a e}+\omega_{a e}\right) \\
& -\frac{1}{3} \Theta a\left(2 B_{a} \dot{u}_{b}+\dot{u}_{a} B_{b}\right) \\
& +a \dot{u}^{e} B_{e}\left(\sigma_{a b}+\omega_{a b}+\frac{1}{3} \Theta h_{a b}\right) \\
& -a h_{a}{ }^{e} R_{e p b q} B^{p} u^{q} .
\end{aligned}
$$

\section{DETAILED DISCUSSION OF APPROXIMATIONS}

In the papers by Tsagas, Maartens and Barrow [16] |14], the cosmic magnetic field is treated as a coherent test field propagating on the background. The largescale magnetic field $B_{a}$ is assumed to be too weak to destroy the spatial isotropy of the background spacetime, which is taken to be a flat Friedman-Robertson- Walker (FRW) model $^{1}$. This is an acceptable physical approximation when the magnetic field density is small compared to the energy density of the fluid i.e. $B^{2} / \rho<<1$. This issue is discussed in 23, where Zel'dovich calculates that $B^{2} / \rho<8 \times 10^{-5}$ if the model is to be applicable during the whole of the radiation dominated era. Furthermore, the isotropy of the microwave background can be used to place strong limits on the magnitude of the magnetic field 24 26].

Other authors have used alternative approaches to the problem of maintaining isotropy in the background in the presence of magnetic fields. Battaner et al. [1] show that a mean magnetic field is incompatible with a RobertsonWalker metric and therefore assume that there is no mean magnetic field on cosmological scales, i.e. $\left\langle B_{a}\right\rangle=0$. However, they include the presence of magnetic fields in smaller cells, with field directions random on larger scales. Thus, although there is no mean magnetic field, the model includes an average magnetic energy density $<B^{2}>\neq 0$. Kim et al. [9] also follow this approach, considering that, at recombination, field directions are randomly oriented on scales smaller than the Hubble radius.

In order to maintain the coupling between magnetic irregularities and energy density perturbations in a straightforward way, a perfectly conducting medium is introduced. Looking at the covariant form of Ohm's law [12], we have, 


$$
J^{a}+J_{b} u^{b} u^{a}=\sigma E^{a}
$$

where $\sigma$ represents the conductivity of the medium. Projecting into the rest space of a fundamental observer yields

$$
J^{<a>}=\sigma E^{a} .
$$

To have a vanishing electric field, while maintaining non zero spatial currents $\left(J^{<a>} \neq 0\right)$, the conductivity of the medium must be infinite, i.e. $\sigma \rightarrow \infty$.

An alternative approach would be to assume a pure magnetic field with no electric field and no spatial currents. This would reduce (13) to

$$
\varepsilon^{a b c} \dot{u}_{b} B_{c}+\operatorname{curl} B^{a}=0,
$$

which is equivalent to

$$
\dot{u}_{[b} B_{c]}+\frac{1}{a} B_{[c b]}=0 .
$$

Although this appears to couple the acceleration to the field gradient, when this relation is inserted into (22), all the magnetic terms cancel out. This effectively decouples the magnetic field from energy density inhomogeneities.

\section{APPROXIMATION SCHEME}

In order to linearize the equations (24), (25) \& 28, we introduce two smallness parameters. The first, $\epsilon_{1}$ is used to measure the extent to which the gauge-invariant variables deviate from zero (their value in a flat FRW universe). The other parameter $\epsilon_{2}$ is a measure of the Alfvén speed $B^{2} / \rho$.

\section{A. Zero- order quantities}

The energy density $\rho$, pressure $p$ and expansion $\Theta$ do not vanish in the background spacetime. The magnetic field, $B_{a}$, is treated as a small test field propagating on the background. It follows that these variables can regarded as zeroth order in our approximation scheme.

\section{B. First-order quantities [in $\epsilon_{1}$ ]}

In order for the metric in some region of spacetime $\mathcal{U}$ to be written in a perturbed FRW form, the following inequalities must hold for the smallness parameter $\epsilon_{1}$ [14,27:

\footnotetext{
${ }^{2}$ In deriving this, $\tilde{\nabla}_{a} w$ and $\tilde{\nabla}_{a} c_{s}^{2}$ are treated as first order in $\epsilon_{1}$, as in 13 .
}

$$
\begin{aligned}
\frac{\sigma}{H}<\epsilon_{1}, \quad \frac{\omega}{H}<\epsilon_{1}, & \frac{\left|E_{a b}\right|}{H^{2}}<\epsilon_{1}, \quad \frac{\left|H_{a b}\right|}{H^{2}}<\epsilon_{1}, \\
\frac{\left|\tilde{\nabla}_{a} \rho\right|}{\rho H}<\epsilon_{1}, & \frac{\left|\tilde{\nabla}_{a} \Theta\right|}{H^{2}}<\epsilon_{1} .
\end{aligned}
$$

where $\left|E_{a b}\right| \equiv\left(E_{a b} E^{a b}\right)^{1 / 2},\left|\tilde{\nabla}_{a} \rho\right| \equiv\left(\tilde{\nabla}_{a} \rho \tilde{\nabla}^{a} \rho\right)^{1 / 2}$ etc.

Tsagas [14] extends this definition to a magnetized universe by arguing that closeness to a spatially flat FRW spacetime is maintained when additional restrictions are imposed as follows:

$$
\frac{\left|\Pi_{a b}\right|}{H^{2}}<\epsilon_{1}, \quad \frac{\left|\tilde{\nabla}_{b} B_{a}\right|}{H\left|B_{a}\right|}<\epsilon_{1} \quad \text { and } \quad \frac{|K|}{H^{2}}<\epsilon_{1} .
$$

In an exact flat FRW spacetime, all quantities of order $\epsilon_{1}$ vanish identically. Thus, $\sigma_{a b}, \omega_{a b}, \dot{u}_{a},\left\{A, A_{a}\right\},\left\{E_{a b}\right.$, $\left.H_{a b}\right\},\left\{\Delta_{a}, \Theta_{a}\right\},\left\{B_{a b}, \mathcal{B}_{a}\right\},\left\{\mathcal{K}_{a}=a^{2} K\right\}$ are all considered to be first order in $\epsilon_{1}$.

Note that although the magnetic field vector $B_{a}$ is considered to be a zeroth order quantity, its magnitude must remain small so that it does not disturb the isotropy of the background. This ensures that the the anisotropic pressure generated by the magnetic field, $\Pi_{a b}$, is negligible in the background, and therefore $\Pi_{a b}$ may also be regarded as first order in $\epsilon_{1}$.

Linearization of the above equatioons is implemented as follows: All terms higher than first order in $\epsilon_{1}$ are dropped, as well as terms higher than first order in the Alfvén parameter $\epsilon_{2}$. Terms like $\epsilon_{1} \epsilon_{2}$ are however kept.

At the end of the calculation, terms first order in $\epsilon_{2}$ are dropped relative to zero order terms in the coefficients of quantities that are first order in $\epsilon_{1}$. This is permissible since the magnetic field is very weak $\left(B^{2} / \rho<<1\right)$. However, this must be done last, otherwise terms may be dropped relative to others which could later vanish.

\section{LINEAR EQUATIONS}

\section{A. Conservation Equations}

Linearization leaves equation (21) unchanged, but slightly modifies the momentum density equation (22):

$$
\rho\left(1+w+\frac{2 B^{2}}{3 \rho}\right) \dot{u}_{a}+Y_{a}-\frac{2}{a} B_{[a b]} B^{b}=0 .
$$

This, together with (23), gives a useful expression for the acceleration vector:

$$
\dot{u}_{a}=\frac{1}{\left(1+w+\frac{2}{3} \frac{B^{2}}{\rho}\right) a}\left(\frac{2}{\rho} B_{[a b]} B^{B}-c_{s}^{2} \Delta_{a}\right) .
$$


It follows that the divergence of the acceleration $A$ is given by 2

$$
A=\frac{1}{\left(1+w+\frac{2}{3} \frac{B^{2}}{\rho}\right)}\left(-\frac{c_{s}^{2}}{a^{2}} \Delta+\frac{B^{2}}{3 \rho} K-\frac{B^{2}}{2 \rho a^{2}} \mathcal{B}\right) .
$$

\section{B. Linearized Propagation Equations}

The linearized propagation equations for the key gauge-invariant quantities are obtained by dropping terms of $\mathcal{O}(2)$ or higher in both linearisation parameters. Note that terms of mixed order $\left(\epsilon_{1} \epsilon_{2}\right)$ and first order in $\epsilon_{2}$ are kept to start with (but may be dropped later).

In these equations, the expansion scalar $\Theta$ is replaced by the equivalent expression involving the Hubble parameter i.e. $\Theta=3 H$. Also, to first order, all projected time derivatives of quantities that are first order in $\epsilon_{1}$ are equal to their normal time derivatives (e.g. $\dot{\Delta}_{<a>}=\dot{\Delta}_{a}$ ).

It follows that the energy density propagation equation (24) becomes:

$$
\begin{aligned}
\dot{\Delta}_{a} & =3 w H \Delta_{a}-(1+w) \Theta_{a} \\
& -\frac{6 H}{\rho} B_{[a b]} B^{b}+\frac{2 a H B^{2}}{\rho} \dot{u}_{a} .
\end{aligned}
$$

The equation for the expansion gradient (25) involves the spatial gradient of the divergence of the acceleration $A_{a}$. This can be determined using expression (37), the identities for commutations between spatial gradients and time derivatives given in Appendix A, and the spatial gradient of the 3 -curvature $K$, given by

$$
a \tilde{\nabla}_{a} K=2 \rho \Delta_{a}+a \mathcal{B}_{a}-4 H \Theta_{a} .
$$

Using these expressions, the evolution of the expansion gradient (to first order in $\epsilon_{1}$ ) is

$$
\begin{aligned}
\dot{\Theta}_{a} & =-2 H \Theta_{a}-\frac{1}{2} \rho \Delta_{a}-\frac{1}{2} a \mathcal{B}_{a}-3 B_{[a b]} B^{b} \\
& -\frac{c_{s}^{2}}{\left(1+w+\frac{2}{3} \frac{B^{2}}{\rho}\right)} \tilde{\nabla}^{2} \Delta_{a}-\frac{a}{2 \rho\left(1+w+\frac{2}{3} \frac{B^{2}}{\rho}\right)} \tilde{\nabla}^{2} \mathcal{B}_{a} \\
& -\left[\frac{6 c_{s}^{2}(1+w)}{\left(1+w+\frac{2}{3} \frac{B^{2}}{\rho}\right)}+\frac{4 B^{2}}{\rho\left(1+w+\frac{2}{3} \frac{B^{2}}{\rho}\right)}\right] a H \tilde{\nabla}^{b} \omega_{a b} \\
& +\frac{2 B^{2}}{3 \rho\left(1+w+\frac{2}{3} \frac{B^{2}}{\rho}\right)} \rho \Delta_{a}+\frac{B^{2}}{3 \rho\left(1+w+\frac{2}{3} \frac{B^{2}}{\rho}\right)} a \mathcal{B}_{a} \\
& -\frac{4 B^{2}}{3 \rho\left(1+w+\frac{2}{3} \frac{B^{2}}{\rho}\right)} H \Theta_{a} .
\end{aligned}
$$

\footnotetext{
${ }^{3}$ This decomposition in analogous to the decomposition of the first covariant derivative of the 4 - velocity: (4).
}

\section{Scalar Equations}

Focusing on the growth, or decay, of density inhomogeneities, the vector field $\Delta_{a}$ contains more information than is necessary. We can extract the required information by considering a local decomposition of the spatial gradient of $\Delta_{a}$ first introduced in $[28]^{3}$ :

$$
\Delta_{a b} \equiv a \tilde{\nabla}_{b} \Delta_{a}=W_{a b}+\Sigma_{a b}+\frac{1}{3} \Delta h_{a b}
$$

where $W_{a b} \equiv \Delta_{[a b]}$ represents rotations of the density gradient $\Delta_{a}, \Sigma_{a b} \equiv \Delta_{(a b)}-\Delta h_{a b}$ describes the variations of $\Delta_{a}$ associated with pancake-or cigar-like structures, and finally $\Delta \equiv \Delta^{a}{ }_{a}=a \tilde{\nabla}^{a} \Delta_{a}$ is related to spherically symmetric gravitational clumping of matter. It is this scalar variable that is important when examining structure formation.

We also need to consider the following complementary scalar variables 13 :

$$
\mathcal{Z} \equiv a \tilde{\nabla}^{a} \Theta_{a}, \quad \mathcal{B} \equiv \frac{a^{2}}{B^{2}} \tilde{\nabla}^{2} B^{2}, \quad \mathcal{K}=a^{2} K
$$

which represent spatial divergences in the expansion gradient, the energy density gradient of the magnetic field, and perturbations in the spatial curvature respectively.

As before, we linearize the scalar propagation equations by dropping terms of order $\epsilon_{1}^{2}$ or $\epsilon_{2}^{2}$, but retain all terms that are first order in $\epsilon_{2}$.

Equations describing the propagation of these scalars are:

$$
\begin{gathered}
\dot{\Delta}=3 w H \Delta-(1+w) \mathcal{Z}+\frac{3 H B^{2}}{2 \rho} \mathcal{B} \\
-\frac{H B^{2}}{\rho} \mathcal{K}-\frac{2 c_{s}^{2} B^{2}}{\rho\left(1+w+\frac{2}{3} \frac{B^{2}}{\rho}\right)} H \Delta \\
\dot{\mathcal{Z}}=-2 H \mathcal{Z}-\frac{\rho}{2} \Delta-\frac{B^{2}}{2} \mathcal{K}+\frac{B^{2}}{4} \mathcal{B}-\frac{c_{s}^{2}}{\left(1+w+\frac{2}{3} \frac{B^{2}}{\rho}\right)} \tilde{\nabla}^{2} \Delta \\
-\frac{B^{2}}{2 \rho\left(1+w+\frac{2}{3} \frac{B^{2}}{\rho}\right)} \tilde{\nabla}^{2} \mathcal{B}+\frac{2 B^{2}}{3 \rho\left(1+w+\frac{2}{3} \frac{B^{2}}{\rho}\right)} \rho \Delta \\
+\frac{B^{2}}{3 \rho\left(1+w+\frac{2}{3} \frac{B^{2}}{\rho}\right)} B^{2} \mathcal{B}-\frac{4}{3} \frac{B^{2}}{\rho\left(1+w+\frac{2}{3} \frac{B^{2}}{\rho}\right)} H \mathcal{Z},
\end{gathered}
$$




$$
\begin{aligned}
\dot{\mathcal{B}}= & -\frac{4}{3} \mathcal{Z}+\frac{4 H c_{s}^{2}}{\left(1+w+\frac{2}{3} \frac{B^{2}}{\rho}\right)} \Delta-\frac{4 H B^{2}}{3 \rho\left(1+w+\frac{2}{3} \frac{B^{2}}{\rho}\right)} \mathcal{K} \\
+ & \frac{2 H B^{2}}{\rho\left(1+w+\frac{2}{3} \frac{B^{2}}{\rho}\right)} \mathcal{B}, \\
\dot{\mathcal{K}}= & \frac{4 H c_{s}^{2}}{\left(1+w+\frac{2}{3} \frac{B^{2}}{\rho}\right)} \Delta+\frac{2 H B^{2}}{\rho\left(1+w+\frac{2}{3} \frac{B^{2}}{\rho}\right)} \mathcal{B} \\
& -\frac{4 H B^{2}}{3 \rho\left(1+w+\frac{2}{3} \frac{B^{2}}{\rho}\right)} \mathcal{K} .
\end{aligned}
$$

\section{Final Linearized System}

Combining equations (43-46), and finally dropping terms of order $\epsilon_{2}$ with respect to zero order quantities, we obtain a second - order differential equation for the scalar energy density perturbations:

$$
\begin{aligned}
\ddot{\Delta} & =-\left(2+3 c_{s}^{2}-6 w\right) H \dot{\Delta}+\frac{1}{2}\left(1-6 c_{s}^{2}+8 w-3 w^{2}\right) \rho \Delta \\
& +c_{s}^{2} \tilde{\nabla}^{2} \Delta-\frac{2 B^{2} H}{\rho(1+w)} \dot{c}_{s}^{2} \Delta-\frac{1}{2}\left(1-3 c_{s}^{2}+2 w\right) \rho c_{a}^{2} \mathcal{B} \\
& +\frac{1}{2} c_{a}^{2} \tilde{\nabla}^{2} \mathcal{B}+\frac{1}{3}\left(2-3 c_{s}^{2}+3 w\right) \rho c_{a}^{2} \mathcal{K} .
\end{aligned}
$$

It is not immediately obvious that the term containing $\dot{c}_{s}^{2}$ is negligible with respect to the other terms involving $\Delta$. In order to clarify this, we can write $\dot{c}_{s}^{2}$ in terms of $w$ by noting

$$
\begin{aligned}
\dot{c}_{s}^{2} & =\frac{d\left(c_{s}^{2}\right)}{d w} \dot{w} \\
& =\frac{4\left(w-c_{s}^{2}\right)}{(1+w)} H .
\end{aligned}
$$

In what follows we will consider the above equations for values of $w \in\left[0, \frac{1}{3}\right]$, but more specifically, we will look at the dust $w=0$ and radiation dominated $w=\frac{1}{3}$ eras. From equation (58) we see that $w=0 \Rightarrow c_{s}^{2}=0$ and that $w=\frac{1}{3} \Rightarrow c_{s}^{2}=\frac{1}{3}$. Thus, the term drops away during both these eras and is negligible at all other times.

Without that term, the second order propagation equation for $\Delta$ becomes

$$
\begin{aligned}
\ddot{\Delta} & =-\left(2+3 c_{s}^{2}-6 w\right) H \dot{\Delta}+\frac{1}{2}\left(1-6 c_{s}^{2}+8 w-3 w^{2}\right) \rho \Delta \\
& +c_{s}^{2} \tilde{\nabla}^{2} \Delta-\frac{1}{2}\left(1-3 c_{s}^{2}+2 w\right) \rho c_{a}^{2} \mathcal{B}+\frac{1}{2} c_{a}^{2} \tilde{\nabla}^{2} \mathcal{B} \\
& +\frac{1}{3}\left(2-3 c_{s}^{2}+3 w\right) \rho c_{a}^{2} \mathcal{K} .
\end{aligned}
$$

\footnotetext{
${ }^{4}$ At this point Tsagas and Maartens 16 expand the LHS of equation (51) using the definition of $\mathcal{K}$ (see equation (42)): $\dot{\mathcal{K}}=2 H \mathcal{K}+a^{2} \dot{K}$ and then drop the second term on the RHS of (51) relative to $2 H \mathcal{K}$.
}

Finally, the completely linearized propagation equations for $\mathcal{B}$ and $\mathcal{K}$ are

$$
\dot{\mathcal{B}}=\frac{4}{3(1+w)} \dot{\Delta}+4 H \frac{\left(c_{s}^{2}-w\right)}{(1+w)} \Delta
$$

and

$$
\dot{\mathcal{K}}=\frac{4 H c_{s}^{2}}{(1+w)} \Delta-\frac{4}{3} \frac{B^{2} H}{\rho(1+w)} \mathcal{K}+\frac{2 B^{2} H}{\rho(1+w)} \mathcal{B} .
$$

Equations (49) and (50) are identical to those in [16], but as a result of slightly different linearisation ${ }^{4}$, equation (51) has an extra term compared to the corresponding result in 16$]$.

\section{DYNAMICAL SYSTEMS ANALYSIS}

Instead of attempting to find exact solutions to equations (49-51) for a dust-radiation background, we instead follow Bruni and Piotrowska [19] and perform a qualitative analysis of the above system of differential equations.

\section{A. The System}

It is useful to change the independent variable from proper time to a function of the scale factor $a$ : with the choice $\tau=\ln a$ (which yields $d \tau / d t=H$ ), and using the standard harmonic decomposition described in Appendix B, equations (49), (50) and (51) become,

$$
\begin{aligned}
\Delta_{(k)}^{\prime \prime} & =-\frac{1}{2}\left(1+6 c_{s}^{2}-15 w\right) \Delta_{(k)}^{\prime}+\frac{3}{2}\left(1-6 c_{s}^{2}+8 w-3 w^{2}\right) \Delta_{(k)} \\
& -c_{s}^{2} \frac{k^{2}}{a^{2} H^{2}} \Delta_{(k)}-\frac{3}{2}\left(1-3 c_{s}^{2}+2 w\right) c_{a}^{2} \mathcal{B}_{(k)}-\frac{c_{a}^{2}}{2} \frac{k^{2}}{a^{2} H^{2}} \mathcal{B}_{(k)} \\
& +\left(2-3 c_{s}^{2}+3 w\right) c_{a}^{2} \mathcal{K}_{(k)}, \\
& \mathcal{B}_{(k)}^{\prime}=\frac{4}{3(1+w)} \Delta_{(k)}^{\prime}+\frac{4\left(c_{s}^{2}-w\right)}{(1+w)} \Delta_{(k)},
\end{aligned}
$$

and

$\mathcal{K}_{(k)}^{\prime}=\frac{4 c_{s}^{2}}{(1+w)} \Delta_{(k)}-\frac{4 c_{a}^{2}}{3(1+w)} \mathcal{K}_{(k)}+\frac{2 c_{a}^{2}}{(1+w)} \mathcal{B}_{(k)}$,

where a prime denotes differentiation with respect to $\tau$. From now on we will drop the subscript $(k)$. 


\section{B. Coefficients in Terms of $w$}

In order to close the system, propagation equations are needed for all background variables. It turns out however that the coefficients of the above differential equations can all be written explicitly as functions of the equation of state parameter $w$, thus only an evolution equation for $w$ needs to be found. To achieve this, we normalise the scale factor $a$ at dust-radiation equi-density by introducing the variable $S=a / a_{E}$ (see Ehlers \& Rindler 29] and Padmanabhan [30]).

Since the two fluids are coupled only through gravity, the energy conservation equation is obeyed separately for each component. This gives $\rho_{d}=\frac{\rho_{E}}{2} S^{-3}$ and $\rho_{r}=\frac{\rho_{E}}{2} S^{-4}$ for dust and radiation respectively, where $\rho_{E}$ is the total energy density at equi - density. Total energy conservation yields $\rho=\frac{\rho_{E}}{2}\left(S^{-3}+S^{-4}\right)$. The only contribution to the total pressure comes from radiation component: $p=p_{r}=\frac{\rho_{r}}{3}=\frac{\rho_{E}}{6} S^{-4}$. It is now straightforward to find $w$ in terms of $S$ [19]:

$$
w=\frac{1}{3(S+1)},
$$

which can be inverted to give

$$
S=\frac{1-3 w}{3 w} .
$$

In this way the expansion of the universe model is parameterised by $w$, with $w \in\left[0, \frac{1}{3}\right]$ and varies from a pure radiation-dominated $(t \rightarrow 0)$ to a pure dust-dominated $(t \rightarrow \infty)$ phase.

These results (55) and (56) allow us to write the coefficients that occur in the perturbation equations as functions of $w$ only (see Appendix Q).

The energy density of the magnetic field $B^{2}$ has a radiation - like propagation equation $B^{2}=B_{E}{ }^{2} S^{-4}$, hence the Alfvén speed can be written as

$$
c_{a}^{2}=6 w c_{a E}^{2} .
$$

The propagation equation for $w$ can now be written down. It is

$$
w^{\prime}=3 w\left(w-\frac{1}{3}\right) \text {. }
$$

In terms of $w$, (52) becomes

$$
\Delta^{\prime \prime}=\alpha_{\Delta} \Delta^{\prime}+\beta_{\Delta} \Delta+\gamma_{\Delta} \mathcal{B}+\eta_{\Delta} \mathcal{K}
$$

where

$$
\begin{aligned}
& \alpha_{\Delta}=-2 \frac{\left(1-3 w^{2}\right)}{(1+w)}+\frac{3}{2}(1+w), \\
& \beta_{\Delta}=\frac{3}{2}\left[1+\frac{w^{2}(5-3 w)}{(1+w)}\right]-\frac{8(1-3 w)^{2}}{9(1+w)} k_{E}^{2}, \\
& \gamma_{\Delta}=-9 w c_{a E}^{2}\left[\frac{1-w+2 w^{2}}{(1+w)}\right]-2 c_{a E}^{2}(1-3 w)^{2} k_{E}^{2}, \\
& \eta_{\Delta}=6 w c_{a E}^{2}\left[\frac{\left(2+w+3 w^{2}\right)}{(1+w)}\right] .
\end{aligned}
$$

Similarly, 53) and (54) become

$$
\mathcal{B}^{\prime}=\alpha_{B} \Delta^{\prime}+\beta_{B} \Delta
$$

and

$$
\mathcal{K}^{\prime}=\alpha_{K} \Delta+\beta_{K} \mathcal{K}+\gamma_{K} \mathcal{B}
$$

where

$$
\begin{aligned}
\alpha_{B} & =\frac{4}{3(1+w)} \\
\beta_{B} & =\frac{4 w}{3(1+w)^{2}}(1-3 w), \\
\alpha_{K} & =\frac{16 w}{3(1+w)^{2}} \\
\beta_{K} & =-\frac{8 w}{(1+w)} c_{a E}^{2} \\
\gamma_{K} & =\frac{12 w}{(1+w)} c_{a E}^{2}
\end{aligned}
$$

with the evolution of the background determined by (58).

\section{Reducing the Order of the System}

In the simplest case of a single fluid, equations (59), (61) and (62) together with the condition $w=$ constant, form a fourth-order autonomous system of differential equations. In the more general two-component case, the system is five dimensional due to the inclusion of the propagation equation for $w(58)$. However, the order of the system can be reduced by introducing a new variable $\mathcal{U}$ [19]. This reduction makes it possible to easily determine the qualitative behaviour of $\Delta$, rather than its exact evolution law. We start by defining $X=\Delta^{\prime}$ and

$$
\begin{gathered}
\mathcal{U}=X / \Delta=\Delta^{\prime} / \Delta, \\
\mathcal{R}=\sqrt{\Delta^{2}+X^{2}} .
\end{gathered}
$$

$\mathcal{R}$ represents an 'amplitude' of the perturbation and it is not directly relevant to our analysis. In order to keep the system dimensionally consistent, we define two new variables,

$$
V=\frac{\mathcal{B}}{\Delta}, \quad \text { and } \quad W=\frac{\mathcal{K}}{\Delta} .
$$

In terms of $\mathcal{U}, V, W$, the equations (59), (61) and (62) become

$$
\begin{gathered}
\mathcal{U}^{\prime}=-\mathcal{U}^{2}+\alpha_{\Delta} \mathcal{U}+\beta_{\Delta}+\gamma_{\Delta} V+\eta_{\Delta} W, \\
V^{\prime}=\alpha_{B} \mathcal{U}+\beta_{B}-V \mathcal{U}, \\
W^{\prime}=\alpha_{K}+\beta_{K} W+\gamma_{K} V-W \mathcal{U},
\end{gathered}
$$

with the coefficients determined by $(60), \&(63)$, together with the propagation equation for $w(58)$. 


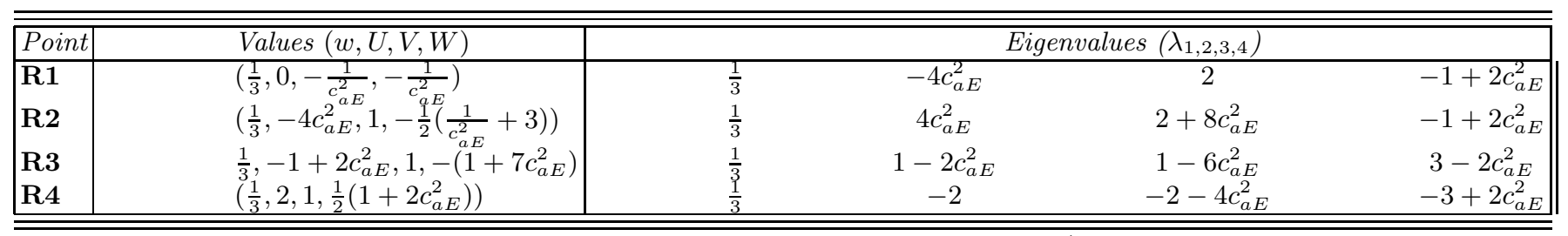

TABLE I. Table of equilibrium points for $w=\frac{1}{3}$

\section{Analysis}

The system is non-linear, however we can analyse it locally by linearising about any stationary points, without losing the details of the qualitative dynamics [31,32].

From equation (58) we can see that stationary points exist for $w=\frac{1}{3}$ and $w=0$.

Since equation (58) decouples from the rest of the system, the 3 -dimensional subsystems corresponding to these values of $w$ correspond to invariant sets describing the early radiation-dominated and late dust-dominated periods of dynamical evolution. We now consider these cases separately.

\section{E. Radiation Era: $w=\frac{1}{3}$}

On substituting $w=\frac{1}{3}$ into $(60)$ and $(63)$, the system for the radiation-dominated era becomes:

$$
\begin{aligned}
\mathcal{U}^{\prime} & =-\mathcal{U}^{2}+\mathcal{U}+2-2 c_{a E}^{2} V+4 c_{a E}^{2} W, \\
V^{\prime} & =\mathcal{U}-V \mathcal{U}, \\
W^{\prime} & =1-2 c_{a E}^{2} W+3 c_{a E}^{2} V-W \mathcal{U} .
\end{aligned}
$$

The equilibrium points for $(69)$ can easily be determined and are given in table $\mathbb{1}$ above.

The 3 - dimensional space described by $\{\mathcal{U}, V, W\}$, and $w=\frac{1}{3}$ (see figure I) is an invariant set, so we can classify the equilibrium points according to the eigenvalues $\lambda_{2,3,4}$ (since $\lambda_{1}=\frac{1}{3}$ is not relevant).

Point R1 is a saddle (not included in figure I because it is far away from the other points). Orbits close to it may initially evolve towards it, but end up evolving away again. At $\mathbf{R} \mathbf{1} \mathcal{U}=0$, so density perturbations neither grow or decay. Using equations (64) and (65) it follows that $\Delta, \mathcal{B}$ and $\mathcal{K}$ are constant:

$$
\begin{aligned}
\Delta & =\text { const }=C_{0}, \\
\mathcal{B} & =-\frac{1}{c_{a E}^{2}} C_{0}, \\
\mathcal{K} & =-\frac{1}{c_{a E}^{2}} C_{0} .
\end{aligned}
$$

Point $\mathbf{R 2}$ is also a saddle, but this time since $\mathcal{U}<0$, density inhomogeneities are decreasing. Solutions at $\mathbf{R 2}$ again follow from equations (64) and (65):

$$
\begin{aligned}
\Delta & =C_{1}\left(\frac{a}{a_{E}}\right)^{-4 c_{a E}^{2}} \\
\mathcal{B} & =\Delta=C_{1}\left(\frac{a}{a_{E}}\right)^{-4 c_{a E}^{2}}, \\
\mathcal{K} & \approx-\frac{1}{2 c_{a E}^{2}} C_{1}\left(\frac{a}{a_{E}}\right)^{-4 c_{a E}^{2}} .
\end{aligned}
$$

Point R3 is a node source, representing unstable equilibrium, so all orbits close to this point evolve away from it. Again since $\mathcal{U}=-1+2 c_{a E}^{2}<0$, solutions at R3 represent a decreasing density inhomogeneity:

$$
\begin{aligned}
\Delta & =C_{2}\left(\frac{a}{a_{E}}\right)^{\left(-1+2 c_{a E}^{2}\right)}, \\
\mathcal{B} & =\Delta=C_{2}\left(\frac{a}{a_{E}}\right)^{\left(-1+2 c_{a E}^{2}\right)} \\
\mathcal{K} & =-\left(1+7 c_{a E}^{2}\right) C_{2}\left(\frac{a}{a_{E}}\right)^{\left(-1+2 c_{a E}^{2}\right)} .
\end{aligned}
$$

Point R4 is a stable node, or a sink. Orbits close to this point evolve towards it as $\left(\frac{a}{a_{E}}\right)$ increases. Since $\mathcal{U}>0$ solutions at $\mathbf{R} \mathbf{4}$ represent growing density inhomogeneities:

$$
\begin{aligned}
\Delta & =C_{3}\left(\frac{a}{a_{E}}\right)^{2}, \\
\mathcal{B} & =\Delta=C_{3}\left(\frac{a}{a_{E}}\right)^{2}, \\
\mathcal{K} & =\frac{1}{2}\left(1+2 c_{a E}^{2}\right) C_{3}\left(\frac{a}{a_{E}}\right)^{2} .
\end{aligned}
$$

Solutions at the points given above correspond to approximate solutions (to leading order in $c_{a E}^{2}$ ) of the perturbation equations during the radiation - dominated era. Therefore by linearity, the general solutions for the perturbation variables $\Delta, \mathcal{B}$ and $\mathcal{K}$ are given by a linear combination of $(70-73)$ :

$$
\begin{aligned}
\Delta & =C_{0}+C_{1}\left(\frac{a}{a_{E}}\right)^{-4 c_{a E}^{2}}+C_{2}\left(\frac{a}{a_{E}}\right)^{\left(-1+2 c_{a E}^{2}\right)}+C_{3}\left(\frac{a}{a_{E}}\right)^{2} \\
\mathcal{B} & =-\frac{1}{c_{a E}^{2}} C_{0}+C_{1}\left(\frac{a}{a_{E}}\right)^{-4 c_{a E}^{2}} \\
& +C_{2}\left(\frac{a}{a_{E}}\right)^{\left(-1+2 c_{a E}^{2}\right)}+C_{3}\left(\frac{a}{a_{E}}\right)^{2} \\
\mathcal{K} & \approx-\frac{1}{c_{a E}^{2}} C_{0}-\frac{1}{2 c_{a E}^{2}}\left(\frac{a}{a_{E}}\right)^{-4 c_{a E}^{2}} \\
& -\left(1+7 c_{a E}^{2}\right) C_{2}\left(\frac{a}{a_{E}}\right)^{\left(-1+2 c_{a E}^{2}\right)} \\
& +\frac{1}{2}\left(1+2 c_{a E}^{2}\right) C_{3}\left(\frac{a}{a_{E}}\right)^{2} .
\end{aligned}
$$

This can be written more concisely as: 


\begin{tabular}{|l|l|cc|}
\hline \hline Point & Values $(w, U, V, W)$ & \multicolumn{2}{c|}{ Eigenvalues $\left(\lambda_{1,2,3,4}\right)$} \\
\hline D1 & $\left(0,0, \frac{1}{2 c_{a E}^{2}}\left[\frac{3}{2 k_{E}^{2}}-\frac{8}{9}\right]\right.$, const $)$ & $-\frac{1}{3}$ & $-\frac{1}{4}+\xi$ \\
D2 & $\left(0,\left[-\frac{1}{4}+\xi\right], \frac{4}{3}, 0\right)$ & $-\frac{1}{3}$ & $\frac{1}{4}-\xi$ \\
D3 & $\left(0,\left[-\frac{1}{4}-\xi\right], \frac{4}{3}, 0\right)$ & $-\frac{1}{3}-\xi$ & $\frac{1}{4}-\xi$ \\
\hline \hline
\end{tabular}

TABLE II. Table of equilibrium points for $w=0$, with $\xi=\frac{1}{2} \sqrt{6\left(\frac{25}{24}-\frac{k_{E}^{2}}{k_{E C}^{2}}\right)}$.

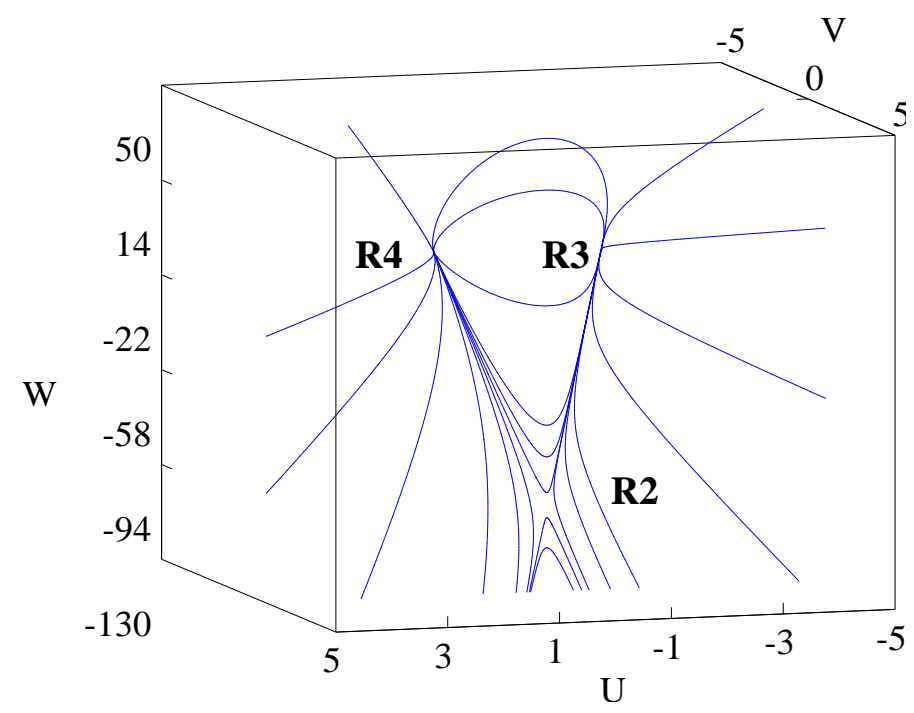

Figure I:

$$
\begin{aligned}
\Delta & =C_{(0)}+\sum_{\alpha} C_{(\alpha)}\left(\frac{a}{a_{E}}\right)^{\alpha}, \\
\mathcal{B} & =-\frac{1}{c_{a E}^{2}} C_{0}+\sum_{\alpha} C_{(\alpha)}\left(\frac{a}{a_{E}}\right)^{\alpha}, \\
\mathcal{K} & =-\frac{1}{c_{a E}^{2}}+\left(1+3 c_{a E}^{2}\right) \sum_{\alpha} \frac{C_{(\alpha)}}{\left(\alpha+2 c_{a E}^{2}\right)}\left(\frac{a}{a_{E}}\right)^{\alpha},
\end{aligned}
$$

where $\alpha$ solves the cubic equation:

$$
\alpha^{3}+\left(2 c_{a E}^{2}-1\right) \alpha^{2}-2 \alpha-8 c_{a E}^{2}\left(1+c_{a E}^{2}\right)=0,
$$

and corresponds to the super - horizon solutions given in [16], with slightly modified exponents - due to the extra term in the equation for the spatial curvature $\mathcal{K}(54)$.

\section{F. Dust Era}

When solving for the equilibrium points in the dustdominated era, we substitute $w=0$ into the equations defining the dynamical system (66-68). This yields:

$$
\begin{aligned}
\mathcal{U}^{\prime} & =-\mathcal{U}^{2}-\frac{1}{2} \mathcal{U}+\frac{3}{2}-\frac{8}{9} k_{E}^{2}-2 c_{a E}^{2} k_{E}^{2} V, \\
V^{\prime} & =\frac{4}{3} \mathcal{U}-V \mathcal{U}, \\
W^{\prime} & =-W \mathcal{U} .
\end{aligned}
$$

The equilibrium points of this system are shown in table II. In this case, the $\mathcal{U}$ and $V$ propagation equations decouple from the equation for $W^{\prime}$, which means that the system is effectively only 2 - dimensional. A critical scale $\lambda_{E c} \equiv 2 \pi a / k_{E c}$ appears in the analysis of these equation through its corresponding wave number $k_{E c}$ :

$$
k_{E c}^{2}=\frac{27}{16} \frac{1}{1+3 c_{a E}^{2}} \approx \frac{27}{16}\left(1-3 c_{a E}^{2}\right) .
$$

The solutions at each point will have different behaviour depending on the value of $k_{E}$. In what follows, we first look at the general properties of the equilibrium points and their corresponding solutions and then give specific information about their behaviour for the three regimes of $k_{E}$ values that emerge from the analysis.

Point D1: These solutions are confined to the $\{\mathcal{U}, V\}$ plane for constant $W$ and approach a constant value independent of the value of $k_{E}$.

$$
\begin{aligned}
\Delta & =\text { const }=C_{0}, \\
\mathcal{B} & =\frac{1}{2 c_{a E}^{2}}\left[\frac{3}{2 k_{E}{ }^{2}}-\frac{8}{9}\right] C_{0}, \\
\mathcal{K} & =\text { const }=C_{k} .
\end{aligned}
$$

Point D2: This point has different stability behaviour for different values of $k_{E}$. The curvature variable $W$ vanishes independently of $k_{E}$. Since the solution at the point depends on $\xi=\xi\left(k_{E}\right)$, the nature of the solution mode (e.g. growing/decaying/oscillation) will depend on the value of $k_{E}$ :

$$
\begin{aligned}
\Delta & =C_{1}\left(\frac{a}{a_{E}}\right)^{\left(-\frac{1}{4}+\xi\right)}, \\
\mathcal{B} & =\frac{4}{3} C_{1}\left(\frac{a}{a_{E}}\right)^{\left(-\frac{1}{4}+\xi\right)} \\
\mathcal{K} & =0 .
\end{aligned}
$$

Point D3: This point has different stability behaviour in two different regions of $k_{E}$. In both regions, the curvature variable vanishes. The solutions at D3 are:

$$
\begin{aligned}
\Delta & =C_{2}\left(\frac{a}{a_{E}}\right)^{\left(-\frac{1}{4}-\xi\right)}, \\
\mathcal{B} & =\frac{4}{3} C_{2}\left(\frac{a}{a_{E}}\right)^{\left(-\frac{1}{4}-\xi\right)}, \\
\mathcal{K} & =0 .
\end{aligned}
$$


As in the radiation dominated era (discussed in section VIIE), solutions (to leading order in $c_{a E}^{2}$ ) for the perturbation variables $\Delta, \mathcal{B}$ and $\mathcal{K}$ are given by a linear combination of the solutions at D1, D2 and D3:

$\Delta=C_{0}+C_{1}\left(\frac{a}{a_{E}}\right)^{\left(-\frac{1}{4}+\xi\right)}+C_{2}\left(\frac{a}{a_{E}}\right)^{\left(-\frac{1}{4}-\xi\right)}$,

$\mathcal{B}=\frac{1}{2 c_{a E}^{2}}\left[\frac{3}{2 k_{E}{ }^{2}}-\frac{8}{9}\right] C_{0}+\frac{4}{3} C_{1}\left(\frac{a}{a_{E}}\right)^{\left(-\frac{1}{4}+\xi\right)}+\frac{4}{3} C_{2}\left(\frac{a}{a_{E}}\right)^{\left(-\frac{1}{4}-\xi\right)}$

$\mathcal{K}=$ const $=C_{k}$.

We now look more closely at how the nature of the stationary points D1, D2 and D3 and their corresponding solutions depends on the wavenumber $k_{E}$.

$\underline{\text { Region } 1-k_{E} \leq k_{E c} . \text { See figure II }}$

Point D1: In this region, the eigenvalues are, respectively, $\lambda_{2}=0, \lambda_{3} \geq 0$, and $\lambda_{4}<0$. It follows that $\mathbf{D 1}$ corresponds to a line of saddles in the solution space for $\{U, V, W\}$.

Point D2: Here, all the eigenvalues are either negative or zero, so this point is a stable sink. Since $\xi \geq \frac{1}{4} \Rightarrow \mathcal{U} \geq 0$, it follows that the solution at D2 corresponds to a growing density inhomogeneity.

Point D3: All eigenvalues are positive, so this point is an unstable node. Since $\mathcal{U}=-\frac{1}{4}-\xi<0$, it follows that the solution at D3 corresponds to a decaying density inhomogeneity.

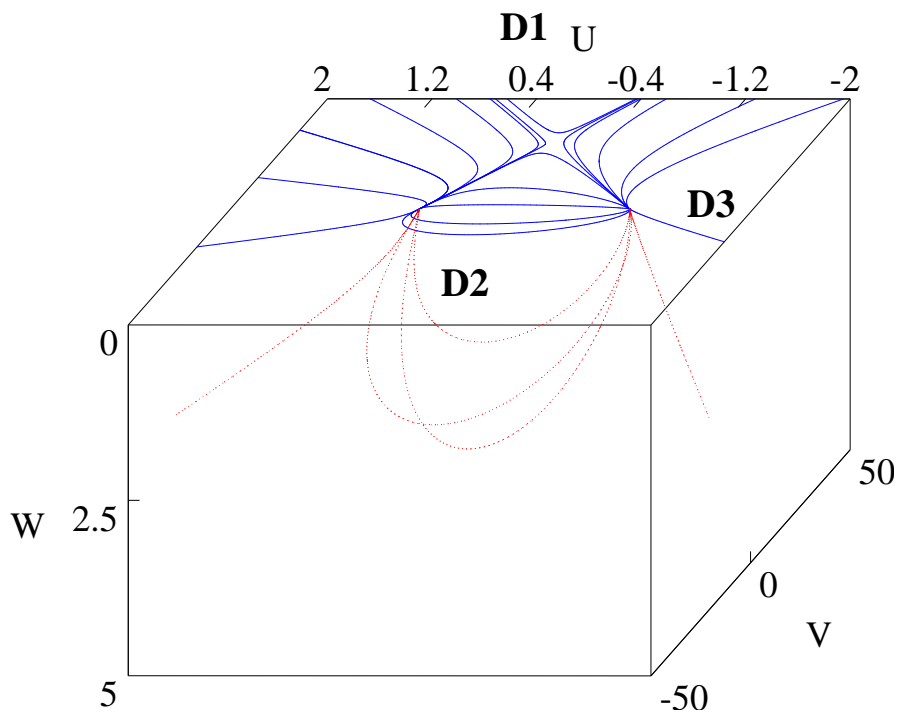

Figure II:

For very long wavelength solutions i.e. $k_{E}<<k_{E C}$ we have $\xi \approx \frac{5}{4}$. In this case the explicit solutions of the perturbation equations (which are a linear combination of the solutions at the points D1, D2 and D3) for the dust era are given by:

$$
\Delta=C_{0}+C_{1}\left(\frac{a}{a_{E}}\right)+C_{2}\left(\frac{a}{a_{E}}\right)^{-\frac{3}{2}}
$$

$$
\begin{aligned}
\mathcal{B} & =\frac{1}{2 c_{a E}^{2}}\left[\frac{3}{2 k_{E}{ }^{2}}-\frac{8}{9}\right] C_{0}+\frac{4}{3} C_{1}\left(\frac{a}{a_{E}}\right)+\frac{4}{3} C_{2}\left(\frac{a}{a_{E}}\right)^{-\frac{3}{2}} \\
\mathcal{K} & =C_{k} .
\end{aligned}
$$

In the dust era, $\left(\frac{a}{a_{E}}\right)=\left(\frac{t}{t_{E}}\right)^{2 / 3}$, so we can write the above solutions (83) in terms of $\left(\frac{t}{t_{E}}\right)$ as in [16]. For example, , the solution for density perturbations is:

$$
\Delta=C_{0}+C_{1}\left(\frac{t}{t_{E}}\right)^{2 / 3}+C_{2}\left(\frac{t}{t_{e}}\right)^{-1}
$$

It consists of a magnetic field induced constant mode, plus the two usual non-magnetized adiabatic modes. Comparing to 16 equation (65), we see that the dynamical systems analysis does not recover the non-adiabatic decaying mode. This is because our analysis is set up to look for asymptotic solutions, so as $t \rightarrow \infty$, the magnetic energy density decays faster than that of the fluid, and $c_{a}^{2} \rightarrow 0$. This removes the magneto-curvature coupling from the propagation equation for $\mathcal{K}$, and therefore our analysis does not obtain the extra decaying mode induced by the magnetic field.

$\underline{\text { Region } 2-k_{E c}<k_{E} \leq \frac{5}{2 \sqrt{6}} k_{E c} \text {. See figure III }}$

Point D1: In this region, the eigenvalues are, respectively, $\lambda_{2}=0, \lambda_{3}<0$ and $\lambda_{4}<0$. It follows that D1 represents a line of sinks (only one is shown in figure III) in the solution space for $\{U, V, W\}$.

Point D2: In this region, the eigenvalues are, respectively $\lambda_{2} \leq 0, \lambda_{3}>0$ and $\lambda_{4}>0$, so D2 is a saddle point (or a line of sources if $\lambda_{2}=0$ ) in the solution space for $\{U, V, W\}$. It follows, since $\mathcal{U}<0$, the solution at $\mathbf{D 2}$ represents a decaying inhomogeneity.

Point D3: Here we obtain the same behaviour as in the case when $k_{E} \leq k_{E C}$, i.e. D3 is a saddle, corresponding to a decaying density inhomogeneity.

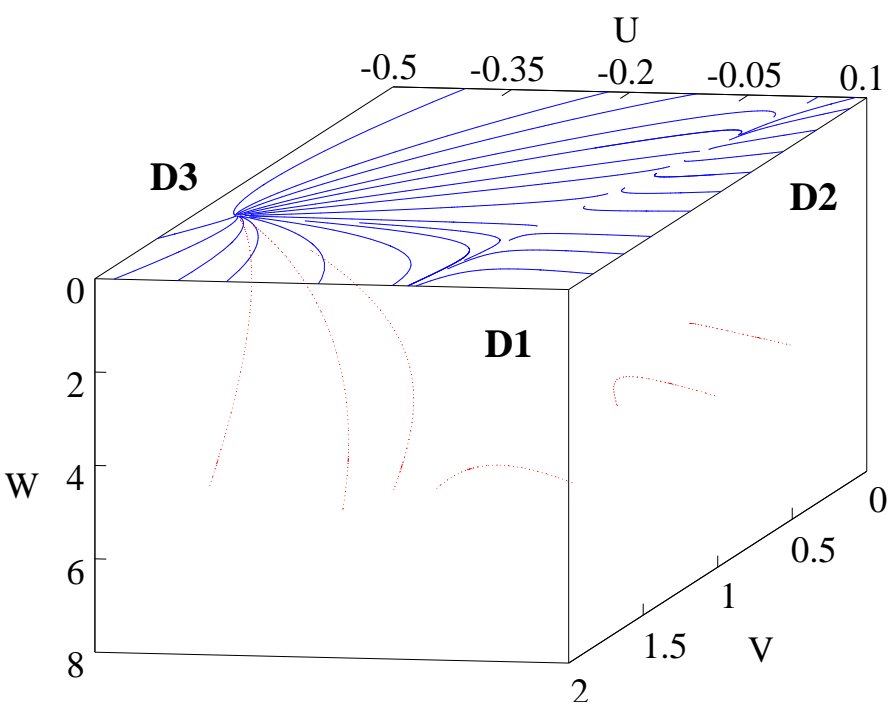




\section{Figure III:}

As for region 1 we can now write down explicit solutions of the perturbation equations. In terms of proper time $\frac{t}{t_{E}}$ we obtain:

$\Delta=C_{0}+C_{1}\left(\frac{t}{t_{E}}\right)^{\left(-\frac{1}{6}+\xi_{t}\right)}+C_{2}\left(\frac{t}{t_{E}}\right)^{\left(-\frac{1}{6}-\xi_{t}\right)}$,

$\mathcal{B}=\frac{1}{2 c_{a E}^{2}}\left[\frac{3}{2 k_{E}{ }^{2}}-\frac{8}{9}\right] C_{0}+\frac{4}{3} C_{1}\left(\frac{t}{t_{E}}\right)^{\left(-\frac{1}{6}+\xi_{t}\right)}+\frac{4}{3} C_{2}\left(\frac{t}{t_{E}}\right)^{\left(-\frac{1}{6}-\xi_{t}\right)}$

$\mathcal{K}=$ const $=C_{k}$,

where

$$
\xi_{t}=\frac{1}{3} \sqrt{6\left(\frac{25}{24}-\frac{k_{E}^{2}}{k_{E C}^{2}}\right)}
$$

and $0 \leq \xi_{t}<\frac{1}{6}$.

In this region, none of the solutions correspond to growing modes for density inhomogeneities. The line of stable sinks represent modes where the density perturbation approaches constant value.

Region $3-k_{E}>\frac{5}{2 \sqrt{6}} k_{E c}$. See figures IV and V

Point D1: For these values of $k_{E}$, the eigenvalues $\lambda_{3}$ and $\lambda_{4}$ are imaginary. Since their real parts are both negative, this solution acts as an attractor, and gives rise to a line of stable spiral points. This repetition of the equilibrium points is, as mentioned before, a consequence of one of the eigenvalues vanishing. Here, the density and magnetic parts of the solution decouple from the curvature ( $W^{\prime}$ equation), and thus the spiral point exists in any $W=$ const plane (see figure $\mathrm{V}$ ).

Point D2: The solutions for $\mathcal{U}$ are imaginary in this region, so density inhomogeneities oscillates as a sound wave, neither growing nor decaying.

Point D3: Here there are no real solutions. Density perturbations again oscillate as sound waves.

This time the solutions are given by

$$
\begin{aligned}
\Delta & =C_{0}+C_{1}\left(\frac{a}{a_{E}}\right)^{\left(-\frac{1}{4}+i \beta\right)}+C_{2}\left(\frac{a}{a_{E}}\right)^{\left(-\frac{1}{4}-i \beta\right)} \\
\mathcal{B} & =\frac{1}{2 c_{a E}^{2}}\left[\frac{3}{2 k_{E}{ }^{2}}-\frac{8}{9}\right] C_{0} \\
& +\frac{4}{3} C_{1}\left(\frac{a}{a_{E}}\right)^{\left(-\frac{1}{4}+i \beta\right)}+\frac{4}{3} C_{2}\left(\frac{a}{a_{E}}\right)^{\left(-\frac{1}{4}-i \beta\right)} \\
\mathcal{K} & =C_{k}
\end{aligned}
$$

with $\beta=\frac{1}{2} \sqrt{6\left(\frac{25}{24}-\frac{k_{E}^{2}}{k_{E C}^{2}}\right)}$. In terms of the proper time $\frac{t}{t_{E}}$ we obtain:

$$
\begin{aligned}
\Delta & =C_{0}+\left(\frac{t}{t_{E}}\right)^{-\frac{1}{6}}\left(C_{3} \cos \left[\frac{2}{3} \beta \ln \left(\frac{t}{t_{E}}\right)\right]+C_{4} \sin \left[\frac{2}{3} \beta \ln \left(\frac{t}{t_{E}}\right)\right]\right), \\
\mathcal{B} & =\frac{1}{2 c_{a E}^{2}}\left[\frac{3}{2 k_{E}}-\frac{8}{9}\right] C_{0} \\
& +\frac{4}{3}\left(\frac{t}{t_{e}}\right)^{-\frac{1}{6}}\left(C_{3} \cos \left[\frac{2}{3} \beta \ln \left(\frac{t}{t_{E}}\right)\right]+C_{4} \sin \left[\frac{2}{3} \beta \ln \left(\frac{t}{t_{E}}\right)\right]\right), \\
\mathcal{K} & =C_{k} .
\end{aligned}
$$

The spiral behaviour round the stable point is evident from the damped oscillatory solution (see figures IV and $\mathrm{V})$.

In this region, the wavelength of solutions falls below a critical wavelength which is related to the Jeans length with a correction due to the magnetic field (see section VII G, below). Thus, these solutions do not result in growing density inhomogeneities, but in general, oscillate as sound waves. The constant density solution due to the magnetic field (corresponding to D1) represents (84the only stable solution in this region, and can be seen as a spiral point in figure $\mathrm{V}$. Note that this is the only equilibrium point in this figure, as the other 2 solutions represent pure sound waves.

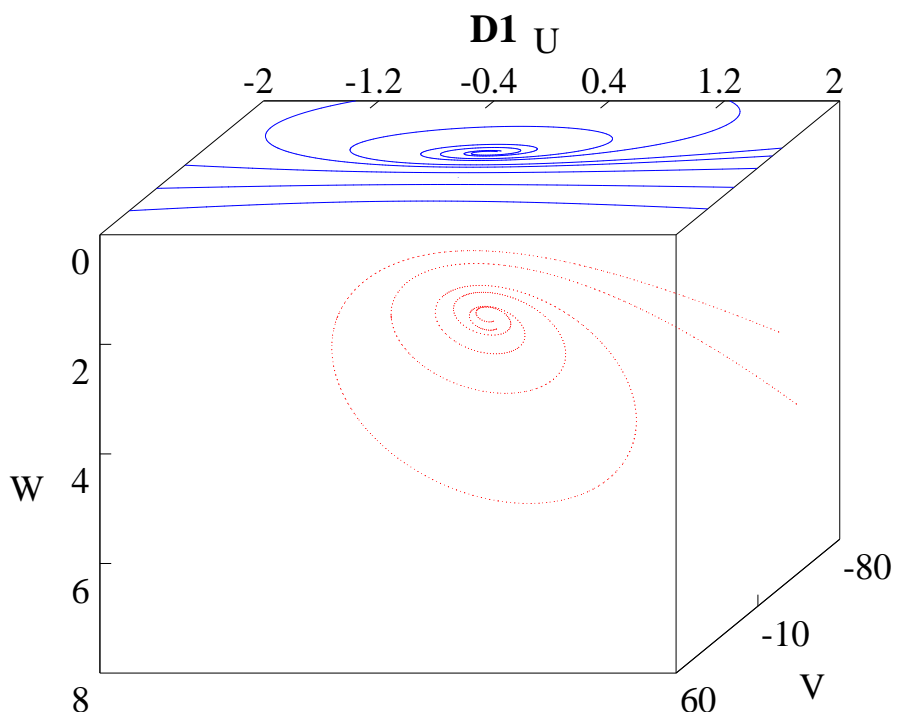

Figure IV:

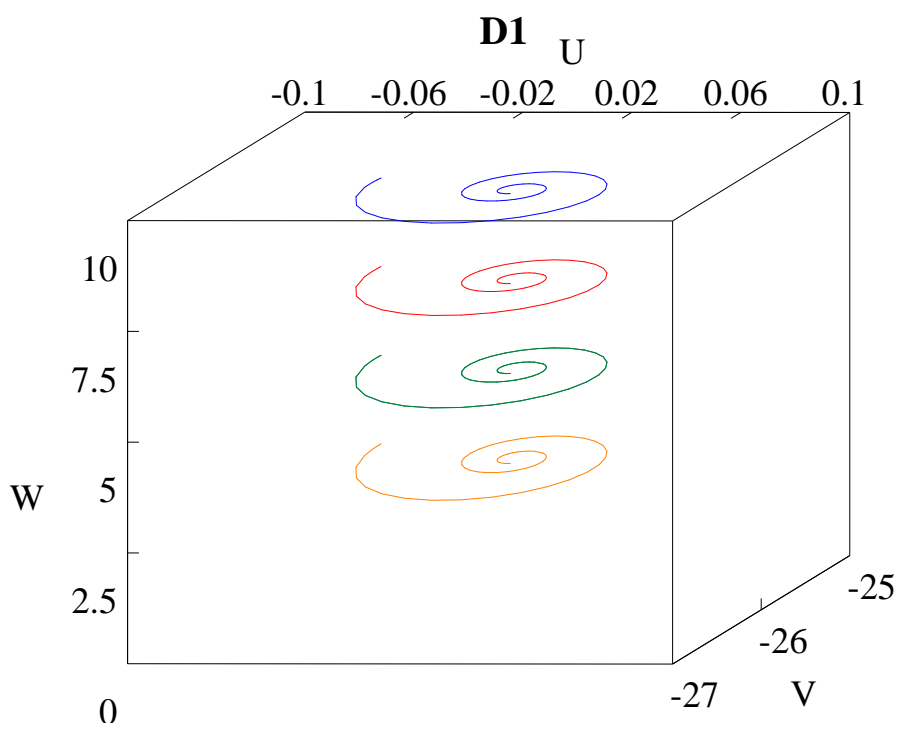


Figure V:

\section{G. Magnetised Jeans length}

It is clears from the above discussions that the critical scale $k_{E C}$ which appears in our analysis is not quite the scale which determines the onset of oscillatory behaviour. This is determined by the wavenumber $k_{o s c}=\frac{5}{2 \sqrt{6}} k_{E C}$, whose corresponding wavelength is:

$$
\begin{aligned}
\lambda_{o s c}=\frac{2 \sqrt{6}}{5} \lambda_{E C} & =\frac{2 \sqrt{6}}{5}\left[\frac{2 \pi}{H_{E}} \frac{4}{3 \sqrt{3}}\left(1+3 c_{a E}^{2}\right)^{1 / 2}\right] \\
& \approx \frac{16 \sqrt{2} \pi}{15} \frac{1}{H_{E}}\left(1+\frac{3}{2} c_{a E}^{2}\right)
\end{aligned}
$$

This scale is closely related to the Jeans Length as it determines the wavelength which divides oscillatory from growing or decaying solutions. It takes the form of a general critical wavelength, modified by a linear factor due to the magnetic field. It is clear that since the magnetic field contributes to the overall pressure, it enlarges the size of regions able to resist gravitational collapse, and so increases the critical scale relative to the non- magnetised case.

In the limiting case $c_{a E}^{2} \rightarrow 0$, we obtain:

$$
\lambda_{o s c} \rightarrow \frac{16 \sqrt{2} \pi}{15} \frac{1}{H_{E}}
$$

which is the corresponding scale for a flat dust - radiation model (with no magnetic field) found by Bruni and Piotrowska in [19].

The scale $\lambda_{o s c}$ found above (88) differs considerably from that found in 16] in their analysis of a magnetized dust solutions (see equation 68). In a pure dust model, the magnetic field acts as an effective pressure, allowing for the extraction of a 'magnetic Jeans length'. This length is directly proportional to the Alfvén speed, and vanishes if there is no magnetic field. Furthermore it is considerably smaller (of order $c_{a E}^{2}$ ) compared to the critical length found by us using a two-fluid model, since in this case, the magnetic field is not the only source of pressure; there is a contribution due to the radiation component.

\section{SUMMARY}

Assuming a large-scale homogeneous magnetic field, we use the exact general relativistic propagation equations given in 12] to describe the evolution of density and magnetic field inhomogeneities and curvature perturbations. We use the usual approximations of small magnetic field energy density $\left(B^{2} / \rho<<1\right)$, and infinite conductivity $(\sigma \rightarrow \infty \Rightarrow E \rightarrow 0)$, to simplify these equations. We carefully linearise the exact equations using a two-parameter approximation scheme and find an extra linear term (compared to [12,13]) in the propagation equation for the spatial curvature $\mathcal{K}$. This extra term however makes no qualitative difference to the dynamics.

Rather than attempting to solve these equations analytically, we follow Bruni and Piotrkowksa [19 by setting up the linear perturbation equations as a fourdimensional autonomous dynamical system. This approach provides an elegant description of the dynamics of gravitational and magnetic inhomogeneities for a two component medium comprising of pressure - free matter (dust) and radiation, interacting only through gravity. The equilibrium points for the three-dimensional invariant sets representing the radiation $\left(w=\frac{1}{3}\right)$ and dust $(w=0)$ eras correspond to the approximate solutions of the perturbation equations derived in Tsagas and Maartens 16. For example in the radiation era we find the following solution for density perturbations on scales much larger than the Hubble radius:

$\Delta=C_{0}+C_{1}\left(\frac{a}{a_{E}}\right)^{-4 c_{a E}^{2}}+C_{2}\left(\frac{a}{a_{E}}\right)^{\left(-1+2 c_{a E}^{2}\right)}+C_{3}\left(\frac{a}{a_{E}}\right)^{2}$.

The adiabatic decaying mode decays even less rapidly due to the magnetic field, and the decaying non-adiabatic mode decays slightly faster than the corresponding solution in [16]. To zeroth order in $c_{a E}^{2}$, these solutions correspond exactly to those given in [16] (see equations (50) and (54) in their paper).

The extra term found in the linearisation of the curvature propagation equation is proportional to $c_{a E}^{2}$, and thus tends asymptotically to zero. Since the dynamical systems analysis examines the asymptotic behaviour of these models, this extra term makes no difference in the analysis of the dust era. Similarly, the superhorion dust solutions do not include the non-adiabatic decaying mode induced by the magnetic field, since the Alfvén speed $\left(c_{a}^{2}=B^{2} / \rho\right)$ also tends to zero at late times.

An important feature that arises out of the dynamical systems analysis, is that we obtain three distinct evolution regimes for the perturbation modes. These regimes are defined through a critical scale $\lambda_{E C}$ which is of the order of the Hubble scale at matter - radiation equidensity and includes a modification linear in $c_{a E}^{2}$ due to the presence of the background magnetic field:

$$
\lambda_{E C}=\frac{1}{H_{E}} \frac{8 \pi}{3 \sqrt{3}}\left(1+3 c_{a E}^{2}\right)^{1 / 2} .
$$

The three regimes are:

- Large wavelengths $-\lambda_{E}>\lambda_{E C}$ These are largescale density and magnetic inhomogeneities that grow unbounded giving gravitational instability. Solutions for these wavelengths consist of a growing mode, a decaying mode, and a constant mode, represented by a saddle point in the phase space of solutions.

- Intermediate wavelengths $-\lambda_{o s c}<\lambda_{E}<\lambda_{E C}$ These perturbations are over-damped and therefore decay asymptotically to a constant value $C_{0}$ without oscillating. 
- Small wavelengths $-\lambda_{E}<\lambda_{o s c}$ Here the perturbations oscillate like sound waves, while their amplitude decays.

Furthermore, the analysis of the dust models yields a magnetic field corrected scale $\lambda_{\text {osc }}$ closely related to the Jeans length, which is a multiple of the general critical scale $\lambda_{E C}$ for flat radiation-dust models:

$$
\begin{aligned}
\lambda_{o s c}= & =\frac{2 \sqrt{6}}{5}\left[\frac{1}{H_{E}} \frac{8 \pi}{3 \sqrt{3}}\left(1+3 c_{a E}^{2}\right)^{1 / 2}\right] \\
& \approx \frac{16 \sqrt{2} \pi}{15} \frac{1}{H_{E}}\left(1+\frac{3}{2} c_{a E}^{2}\right)
\end{aligned}
$$

where the quantities $c_{a E}^{2}, H_{E}, \lambda_{E}$ are evaluated at equidensity.

Finally we note that $\lambda_{\text {osc }}$ is more general than the 'magnetised Jeans Length' found in [16] for a pure dust model, since it takes into account the pressure effects resulting from a proper two-fluid description.

\section{ACKNOWLEDGMENTS}

We thank Mattias Marklund, Christos Tsagas and Roy Maartens for helpful discussions, the referee for his/her comments and the NRF (South Africa) for financial support.

\section{APPENDIX A: COVARIANT IDENTITIES}

This identities are written using the notation of 21] and [16], except using $\tilde{\nabla}$ instead of $D$ for the orthogonally projected covariant derivative. These identities are used in deriving the propagation equations (assuming a flat background and vanishing cosmological constant):

$$
\begin{aligned}
\operatorname{curl} \tilde{\nabla}_{a} f & =-2 \dot{f} \omega_{a}, \\
\left(a \tilde{\nabla}_{a} f\right)^{\cdot} & =a \tilde{\nabla}_{a} \dot{f}+a \dot{f} A_{a}, \\
\tilde{\nabla}^{2}\left(\tilde{\nabla}_{a} f\right) & =\tilde{\nabla}_{a}\left(\tilde{\nabla}^{2} f\right)+2 \dot{f} \operatorname{curl} \omega_{a}, \\
\left(a \tilde{\nabla}_{a} J_{b} . \cdot\right)^{\prime} & =a \tilde{\nabla}_{a} \dot{J}_{b} \ldots, \\
\tilde{\nabla}_{[a} \tilde{\nabla}_{b]} V_{c} & =0=\tilde{\nabla}_{[a} \tilde{\nabla}_{b]} S^{c d}, \\
\operatorname{div} \operatorname{curl} V & =0, \\
(\operatorname{div} \operatorname{curl} S)_{a} & =\frac{1}{2} \operatorname{curl}(\operatorname{div} S)_{a}, \\
\operatorname{curl} \operatorname{curl} V_{a} & =\tilde{\nabla}_{a}(\operatorname{div} V)-\tilde{\nabla}^{2} V_{a} \\
\operatorname{curl} \operatorname{curl} S_{a b} & =\frac{3}{2} \tilde{\nabla}_{\langle a}(\operatorname{div} S)_{b\rangle}-\tilde{\nabla}^{2} S_{a b},
\end{aligned}
$$

where the vectors and tensors vanish in the background and $S_{a b}=S_{\langle a b\rangle}$. The magnetic field vector does not vanish in the background, and so its projected derivatives do not commute to linear order. For the magnetic field, the vector identity in (A5) must therefore be changed to:

$$
\tilde{\nabla}_{[a} \tilde{\nabla}_{b]} B_{c}=\frac{1}{2} \mathcal{R}_{d c b a} B^{d}-\varepsilon_{a b d} \omega^{d} \dot{B}_{c},
$$

where $\mathcal{R}_{a b c d}$ is the 3 - Curvature tensor formed from $R_{a b c d}$ and the kinematic quantities [12,13].

\section{APPENDIX B: HARMONIC DECOMPOSITION}

When setting up and analysing the dynamical system, all the equations can be reduced to ordinary differential equations if we restrict our attention to the harmonic components of the perturbation variables. This is a way of effectively separating the time from the space variables, and involves writing the perturbation scalars in terms of harmonic scalars as follows [14]:

$$
\begin{aligned}
\Delta & =\sum_{n} \Delta_{(n)} Q_{(n)}, \\
\mathcal{B} & =\sum_{n} \mathcal{B}_{(n)} Q_{(n)}, \\
\mathcal{K} & =\sum_{n} \mathcal{K}_{(n)} Q_{(n)} .
\end{aligned}
$$

The scalar harmonics, $Q$, are defined by

$$
\begin{aligned}
\dot{Q}_{(n)} & =0 \quad \text { and } \\
\tilde{\nabla}^{2} Q_{(n)} & =-\frac{n^{2}}{a^{2}} Q_{(n)},
\end{aligned}
$$

where $n=k \geq 0$ (since we are dealing with a flat model). Spatial flatness of the background also means that $k$ is simply related to the wavelength $\lambda$ of the perturbation since $\lambda=\frac{2 \pi a}{k}$.

\section{APPENDIX C: COEFFICIENTS IN TERMS OF $W$}

$c_{s}^{2}$ is only formally the speed of sound since the fluids interact only through gravity. Using the energy-density conservation equations for matter and radiation we can express $c_{s}^{2}$ in terms of the parameter $w$ :

$$
c_{s}^{2}=\frac{\dot{p}}{\dot{\rho}}=\frac{4 w}{3(1+w)} .
$$

The total energy density of the matter - radiation mixture is given by:

$$
\rho=\frac{27}{2} \rho_{E} \frac{w^{3}}{(1-3 w)^{4}}
$$

where $\rho_{E}$ is the energy density at matter-radiation equality. This leads to a similar equation for the Hubble parameter $H^{2}$ :

$$
H^{2}=\frac{27}{2} H_{E}^{2} \frac{w^{3}}{(1-3 w)^{4}}
$$


where $H_{E}$ is the value of the Hubble parameter at matter - radiation equality.

When harmonically decomposing the perturbation equations in section $\mathrm{VII}$, the Laplacian terms give rise to coefficients of the form $k^{2} / a^{2} H^{2}$. These can be expressed in terms of the variable $w$ as follows:

$$
\begin{aligned}
\frac{k^{2}}{a^{2} H^{2}}= & \frac{k^{2}}{a_{E}^{2} H_{E}^{2}} \frac{2(1-3 w)^{2}}{3 w} \\
& =k_{E}^{2} \cdot \frac{2(1-3 w)^{2}}{3 w}
\end{aligned}
$$

where $k_{E}^{2}=\frac{k^{2}}{a_{E}^{2} H_{E}^{2}}$.

[1] E. Battaner \& H. Lesch, On the Physics of Primordial Magnetic Fields, astro-ph/0003370 (2000).

[2] J. G. Bolton and P. R. Wild, Ap. J. 125, 296 (1957).

[3] P. P. Kronberg, Rep. Prog. Phys 57, 325 (1994).

[4] R. Wielebinski, The Interstellar Medium in Galaxies, p. 349, (Dordrecht: Kluwer, 1990)

[5] P. P. Kronberg, Nature 374, 404 (1995).

[6] G. B. Taylor and R. A. Perely, Ap. J. 416, 554 (1993).

[7] T. V. Ruzmaikina \& A. A. Ruzmaikin, Soviet Astronomy - AJ 14, no.6 (1971).

[8] I. Wasserman, Ap. J 224, 337 (1978).

[9] E. Kim, A. Olinto \& R. Rosner, Ap. J 468, 28 (1996).

[10] E. Battaner, E. Florido \& J. Jiménez-Vicente, Astron. Astrophys 326, 13 (1997).

[11] K. Jedamzik, V. Katalinic \& A. Olinto, Phys. Rev D. 57, 3265 (1998). K. Subramanian \& J. D. Barrow, Phys. Rev. D 58, 83502 (1998).
[12] C. G. Tsagas \& J. D. Barrow, Class. Quantum Grav. 14, 2539 (1997).

[13] C. G. Tsagas \& J. D. Barrow, Class. Quantum Grav 15, 3523 (1998).

[14] C. G. Tsagas, PhD Thesis (Sussex University, 1998).

[15] G. F. R. Ellis \& M. Bruni, Phys. Rev. D 54, 1804 (1989).

[16] C. G. Tsagas \& R. Maartens, Phys. Rev. D 61, 083519 (2000).

[17] A. Waszczyna, Phys. Rev. D 45, 1982 (1992).

[18] M. Bruni, Phys. Rev. D 47, 738 (1993).

[19] M. Bruni \& K. Piotrowksa, Mon. Not. R. Astron. Soc. 270, 630 - 640 (1994).

[20] G. F. R. Ellis and H. van Elst, Cosmological models (Cargèse lectures 1998), in Theoretical and Observational Cosmology, edited by M. Lachièze-Rey, p. 1 (Kluwer, Dordrecht, 1999).

[21] R. Maartens, Phys. Rev. D 55, 463-467 (1997).

[22] P. K. S. Dunsby, M. Bruni \& G. F. R. Ellis, Ap. J 395, 54 (1992).

[23] Ya. B. Zel'dovich, Sov. Astron 13, 608 (1970).

[24] J. Adams et. al., Phys. Lett. B 388, 253 (1996).

[25] J. D. Barrow, Phys Rev D 55, 7451 (1997).

[26] J. D. Barrow, P. G. Ferreira \& J. Silk, Phys. Rev. Lett. 78, 3610 (1997).

[27] W. Stoeger, R. Maartens \& G. F. R. Ellis, Astrophys. J 443, 1 - 5 (1995).

[28] G. F. R. Ellis, M. Bruni \& J. Hwang, Phys Rev D 42, $1035-1046$ (1990).

[29] J. Ehlers \& W. Rindler, Mon. Not. R. Astron. Soc. 238, 503 (1989).

[30] T. Padmanabhan, Structure Formation in the Universe, Cambridge University Press (Cambridge, 1993).

[31] U. Nilsson, PhD Thesis (Stockholm University, 1998).

[32] J. Wainwright \& G. F. R. Ellis, Dynamical Systems in Cosmology, Cambridge University Press, (Cambridge, 1997). 